\title{
En el aniversario de Erving Goffman
}

(1922-1982)

Blanca Lozano Maneiro

Universidad Complutense de Madrid

\section{RESUMEN}

Aunque la obra de Erving Goffman goza todavía de escasa implantación en la sociología académica española, es indudable que es uno de los microsociólogos más importantes y originales de la segunda mitad del siglo xx. Por eso, al cumplirse veinte años de su muerte y ochenta de su nacimiento, he tratado de ofrecer una breve revisión bio-bibliográfica de su vida y su pensamiento. Formado en la Escuela Interaccionista Simbólica y creador del Modelo Dramatúrgico, sus intereses científicos le llevan a analizar áreas de investigación como la interacción en la vida cotidiana, la construcción social del estigma, la identidad y el sí-mismo, las Instituciones Totales, etc., que continúan con plena vigencia en el núcleo de las actuales discusiones psicosociológicas.

Palabras clave: Teoría Sociológica, Interacción Social, Psicología Social. 
El 20 de noviembre de 2002 se cumplieron veinte años de la muerte de uno de los microsociólogos más fecundos y originales de la segunda mitad del siglo pasado: Erving Goffman.

Formado en la Escuela de Chicago con interaccionistas simbólicos de la talla de Herbert Blumer y E. C. Hughes, Goffman es el creador del modelo sociológico conocido como «Dramaturgia Social» que, junto con «la Etnometodología» de Harold Garfinfel y «la Teoría del Intercambio Social» de George Homans, surge como intento de salvar a la sociología de la crisis en la que se hallaba sumergida hacía años.

Según todos sus biógrafos, Erving Goffman nace el 11 de junio de 1922 en un lugar de la provincia de Alberta llamado Manville, en Canadá. Hijo de judíos ucranianos emigrados, Goffman pasa su infancia y su primera adolescencia en Dauphine, al norte de Winnipeg, donde sus padres poseían un pequeño comercio.

Goffman estudia el bachillerato en Winnipeg, licenciándose en Sociología en la Universidad de Toronto en junio de 1945. Debe decidir entonces dónde continuar sus estudios. Y elige, influido por su profesor Ray Birdwhistell y por su amiga Liz Bott, la Universidad de Chicago. Allí, los primeros profesores de Goffman son sus propios compañeros de clase. Saul Mendlovitz, uno de sus mejores interlocutores, está fascinado por el gran fenomenólogo husserliano Gustav Ichheiser. Mendlovitz ha leído todo lo que ha escrito y se lo ha pasado a Goffman. Parece claro que el largo artículo de Ichheiser, publicado en 1949 en un suplemento del American Journal of Sociology, titulado «Los Equívocos en las Relaciones Humanas» es uno de los principales textos inspiradores del pensamiento goffmaniano. Goffman, a su vez, está encantado con Kenneth Burke y ha devorado sus libros: Permanence and Change, publicado en 1935, y A Grammar of Motives, publicado en 1945. Probablemente, en ellos atisbó el modelo «dramático» de las relaciones hu-manas.

Además, el mismo Departamento de Sociología de Chicago era en aquella época el «no va más» en los Estados Unidos. Cualquier estudiante que entraba en el Social Science Re-search Building sabía que todos los grandes de la sociología norteamericana, desde William Thomas hasta Robert Park, habían estado allí y cualquiera podía encontrarse por los pasillos con un Wirth, un Blumer o un Hughes. Para colmo, la primera revista estadounidense de sociología, el American Journal of Sociology, se editaba, desde su fundación, por este Departamento.

A finales de 1949, Goffman termina su tesina de licenciatura (Master of Arts), titulada Características de la Reacción a la Experiencia Figurada. Es el primer escrito que conocemos de Goffman y en un principio se trataba, simplemente, de ampliar una investigación sobre 
las reacciones de los oyentes a un serial radiofónico que la CBS (Columbia Broadcasting System) había encargado a Lloyd Warner y a William Henry.

En ese año, Goffman es contratado por la Universidad de Edimburgo como profesor ayudante del recién creado Departamento de Antropología Social. Allí conoce al sociólogo inglés Tom Burns, que está construyendo una teoría sobre las «relaciones de broma» que tiene fascinado a Goffman y sobre la que pensará cuando pasea por una de las islas de las Shetland, quizá la de Unst, que será la elegida para su tesis doctoral. Un día de diciembre de 1949, Goffman llega en una barca a Dixon, la capital de la isla más septentrional del archipiélago de las Shetland.

Finalizado su trabajo de campo, Goffman se va de Dixon en mayo de 1951 y se instala en París para redactar su tesis.

Cuando, a finales de 1951, vuelve a Chicago se encuentra con un Departamento en crisis. Wirth ha muerto de un infarto, Ogburn y Faris están a punto de jubilarse y Blumer se ha ido a Berkeley. Sólo quedan Burgess, Warner y Hughes. Unos meses más tarde, en julio de 1952, Goffman se casa con Angelica Schuyler Choate y un año más tarde, en la primavera de 1953, defiende su tesis doctoral, titulada Conducta comunicativa en una comunidad isleña, obteniendo así el grado de Doctor en Sociología.

El siguiente paso de Goffman es irse al Hospital Saint-Elizabeth, de Washington, a vivir entre los orates. En ese momento, Estados Unidos tiene hospitalizados más de medio millón de enfermos mentales y esta cifra aumenta constantemente. El Instituto Nacional de Salud Mental había pedido al sociólogo John Clausen que organizara un «laboratorio de estudios socioambientales" y Goffman es contratado sobre la base del enfoque que propone. Goffman quiere hacer una investigación desde dentro de la vida hospitalaria. Escribe: «Yo creía, y sigo creyendo, que no hay grupo - trátese de presos, de primitivos, de dotaciones de buques o de enfermos - en el que no se desarrolle una vida propia, que se hace significante, sensata y normal en cuanto se la conoce desde dentro; y que un buen modo de aprender algo sobre cualquiera de esos mundos consiste en someterse personalmente, en compañía de sus miembros, a la rutina diaria de las menudas contingencias a la que ellos mismos están sujetos» (Goffman, 1961: 9).

Así, en el verano de 1954, Goffman, su mujer y su hijo muy pequeño, Tom, dejan Chicago y se van a vivir a Bethesda, en Maryland, donde pasan dos meses.

En 1955 decide estar un año en el St. Elizabeth, un inmenso manicomio de más de 7.000 camas situado en Washington. Dice en el prólogo de Internados: «El objetivo inmediato de 
mi trabajo de campo en el Hospital Saint-Elizabeth, fue tratar de aprender algo sobre el mundo social de los pacientes hospitalizados, tal como ellos lo experimentan subjetivamente» (Goffman, 1961: 9). Así, vestido como un paciente, comiendo con ellos y tratando con ellos irá, durante un año, de un pabellón a otro. Sin embargo, como dice Winkin, es muy posible que saliese conmovido de esa experiencia, pero su sentimiento se manifestará en forma de rabia fría, «con los caracteres de un juicio en el que se mezclan el humor negro, la crítica social y la teoría sociológica» (Winkin, 1991: 78). En octubre de 1956, su antiguo profesor de Toronto, Ray Birdwhistell, le invita a exponer los datos que ha obtenido en sus observaciones en el hospital psiquiátrico a una reunión que todos los años organiza la prestigiosa Fundación Macy para hablar de «procesos de grupo». Entre los asistentes hay psiquiatras y sociólogos de la talla de Gregory Bateson y Margaret Mead y, al parecer, hubo bronca, por supuesto dialéctica, porque «estamos entre gente bien», pero fue increíble la violencia de las palabras. Goffman habla en términos muy duros, haciendo patente su indignación hacia esa auténtica destructora de hombres que es la institución psiquiátrica en los Estados Unidos de los años cincuenta. Ni los psiquiatras ni Margaret Mead lo entienden (sí, Gregory Bateson) y obligan a Goffman, de una forma bastante agresiva, a retractarse. La aversión de éste a la psiquiatría institucional está reforzada por una tragedia que le afecta muy íntimamente: su mujer, Sky, tiene una salud mental muy frágil y sus repetidos intentos de suicidio exigen una constante atención psiquiátrica. Goffman lo admite mal y, en este triste y dramático entorno marital, escribe Internados.

Goffman no sólo introduce el concepto de institución total, sino que realiza el primer análisis orgánico de tales instituciones, y especifica: «Cabe definir una organización instrumental formal como un sistema de actividades deliberadamente coordinadas, que tienden a la obtención de algunos fines generales explícitos» (Goffman, 1961: 177), es decir, la organización como tensión estructurada y sistemática dirigida hacia unos objetivos determinados. Las instituciones totales «son lugares de residencia y trabajo, donde un gran número de individuos en situación análoga, aislados de la sociedad por un período de tiempo, comparten en su encierro un modo de vida administrado formalmente» (Goffman, 1961: 13).

Las instituciones totales absorben parte del tiempo y del interés de los individuos que están en ellas y les proveen de un mundo propio; es decir, tienen tendencias totalizadoras. La tendencia absorbente o totalizadora está representada simbólicamente por los obstáculos que impiden la interacción social con el exterior y la huida de los individuos. Estos obstáculos tienen, en general, forma material: puertas cerradas, altos muros, alambres de espino, acantilados, ríos, bosques o pantanos.

Además, la institución total concede atribuciones concretas de identidad a los individuos que forman parte de ella; es decir, el cuartel transforma al civil en soldado, el convento lo 
transforma en monje y la cárcel en preso. La característica principal de la institución total es su clausura, que es, además, la forma que tienen las relaciones entre este tipo de organizaciones y su contexto social.

En 1956, el Centro de Investigaciones en Ciencias Sociales de la Universidad de Edimburgo edita La presentación de la persona en la vida cotidiana. Este libro, y en concreto la utilización del lenguaje teatral, hará famoso a Goffman y permitirá que sea conocido como el primer representante del análisis «dramatúrgico», aunque él diga, constantemente, que no ha inventado nada y que se ha basado por entero en el «modelo dramatístico» de Kenneth Burke. Como continuador de George Herbert Mead, Goffman está convencido de que el concepto que el individuo tiene de sí mismo surge de la interacción social de las situaciones que componen la vida cotidiana. La comprensión de la conducta social del individuo se alcanza mediante un examen detallado de los ambientes situacionales a los que está respondiendo con el fin de entender la composición de su yo social y las opciones de que pueda disponer para mantener su propia estimación y la ajena; es decir, mediante un análisis de la interacción en la vida cotidiana. Veamos, pues, qué es, para Erving Goffman, la interacción.

Los elementos o unidades de análisis que nos permitirán definir la interacción en cuanto tal son los «encuentros», las «situaciones» y las «ocasiones» sociales. Los individuos, «unidades de interacción», se mezclan constantemente de forma casual y espontánea durante sus actividades en la vida cotidiana formando una trama continua de interacciones, "corresponden plenamente a la gestión de la co-presencia», constituyendo para Goffman «... la unidad fundamental de la vida pública» (Goffman, 1971: 37). Por tanto, sólo estudiando con detalle los encuentros sociales pueden establecerse las normas que los individuos de forma inconsciente siguen al entrar en contacto con los demás. Para Goffman, el examen sistemático y cualitativo de estas «pequeñas conductas» tiene dos objetivos claros:

1. «... Describir las unidades naturales de interacción, a partir de la más pequeña — por ejemplo, el fugaz movimiento facial que puede hacer un individuo».

2. «... Descubrir el orden normativo que predomina en y entre dichas unidades, es decir, el orden conductual que se encuentra en todos los lugares poblados, ya sean públicos, semipúblicos o privados» (Goffman, 1967: 11-12).

Como ya he dicho, Goffman distingue tres unidades conceptuales básicas para el análisis de la interacción:

1. «La situación social», que define como «cualquier ambiente determinado por la posibilidad de un control recíproco tal que pueda prolongarse todo el tiempo que dos o más suje- 
tos se encuentran en inmediata presencia física uno de otro y que se extiende a todo el espacio en el cual semejante control es posible» (Goffman, 1967: 150).

2. «La ocasión social», definida como «un acontecimiento, por ejemplo una cena, que se espera con ansiedad y se contempla retrospectivamente como una unidad; tiene un lugar y un tiempo de desarrollo, y establece el tono de lo que sucede en y durante ella» (Goffman, 1967: 129). Estas ocasiones sociales o de sociabilidad se dividen en dos tipos. El primero se establece por las invitaciones explícitas: fiestas, reuniones, comidas, té, visitas, cócteles, etc. En estos casos, las personas entran en contacto para mantener una conversación sociable que dura un período de tiempo definido. El segundo tipo se manifiesta por los «encuentros fortuitos» entre personas que se han definido recíprocamente «conocidas». Esto significa que entre ellos se ha estipulado, implícita o explícitamente, una especie de contrato a largo plazo que prevé el hecho de que se dará vida a una conversación cada vez que un individuo se encuentre con otro, a menos que no intervengan circunstancias eximentes privadas o públicas.

3. «El encuentro social», por último, «es una ocasión de interacción cara a cara que comienza cuando los individuos reconocen que se han colocado en presencia inmediata unos de otros y que termina con una separación aceptada de la participación mutua. Los encuentros difieren notoriamente en cuanto a sus propósitos, función social, tipo y número de personas, marcos, etc.» (Goffman, 1967: 91).

En las interacciones, la co-presencia exige el establecimiento de un pacto de colaboración necesario para sostener el mayor tiempo posible un centro de atención cognoscitiva, discursiva y visual en el que participen todos los sujetos. Está claro que estos episodios interactivos se crean y cesan dentro de un conjunto de formas sociales institucionalizadas y de relaciones de poder que están por encima de la eventualidad de tales episodios; y hay que señalar que Goffman ha sido acusado de reducir todo el orden social a los encuentros. Él afirma claramente (quizá de forma un tanto cínica) que el objeto fundamental de la sociología es el de la organización y estructura social, y reconoce que no lo toca en sus trabajos. Desde su punto de vista, la estratificación es una realidad sujeta a continuas negociaciones y las etiquetas «clase alta», "clase media» y «clase baja» no son sino términos históricamente relativos. Por ello centra sus estudios en la noción que tiene el individuo de la estructura y la realidad social, en los «residuos cotidianos», lo que, desde luego, no supone ningún derrumbamiento del orden de las cuestiones importantes. Debemos añadir que el hecho de considerar como unidades básicas de la vida cotidiana las interacciones no quiere decir que ignore que en la realidad social actúan, a niveles diferentes, otros supuestos. No podemos encontrar en la obra de Goffman el análisis tradicional del poder en términos macrosociales porque el tipo de poder que él estudia tiene formas propias de fun- 
cionamiento; porque el poder que Goffman analiza es, por ejemplo, el que existe en cualquier pareja, en la familia o el que detenta, momentáneamente, el que tiene el uso de la palabra en cualquier interacción conversacional. Por eso, su análisis se basa fundamentalmente en una dimensión de la organización de la vida social cotidiana, hasta ahora desatendida.

La situación, la ocasión y el encuentro social son auténticos microsistemas sociales, pequeñas realidades sociales que mantienen las personas cuando están juntas. Los encuentros sociales difieren en gran medida de la importancia que les concedan los sujetos, pero, cruciales o no, todos los encuentros son ocasiones en que los individuos pueden estar involucrados de forma espontánea en las actividades y extraer de ello un firme sentimiento de realidad, «y este sentimiento no es trivial, cualquiera que sea el contexto en el cual esté contenido» (Goffman, 1967: 122). Berger y Luckmann entendieron perfectamente la importancia del encuentro como creador de un sentimiento de realidad social. "La experiencia más importante que tengo de los otros se produce en la situación "cara a cara", que es el prototipo de la interacción social y del que se derivan todos los demás casos» (Berger y Luckmann, 1968: 46). Por tanto, la construcción social de la realidad se desarrolla y «"es aprehendida" en un continuum de tipificaciones que se vuelven progresivamente anónimas a medida que se alejan del "aquí y ahora" de la situación "cara a cara". En un polo del continuum están esos otros con quienes me trato a menudo e interactúo intensamente en situaciones "cara a cara", mi "círculo íntimo", diríamos. En el otro polo hay abstracciones sumamente anónimas, que por su misma naturaleza nunca pueden ser accesibles en la interacción "cara a cara". La estructura social es la suma total de estas tipificaciones y de las pautas recurrentes de interacción establecidas por intermedio de ellas. En ese carácter la estructura social es un elemento esencial de la realidad de la vida cotidiana» (Berger y Luckmann, 1968: 51-52).

La interacción cara a cara no sólo expresa un orden social, sino que transforma un mero agregado de personas en un pequeño grupo social con una realidad propia. Su estructura ordena la comunicación sobre el hecho de comunicar y, puesto que cambia a los sujetos participantes en personas mutuamente accesibles a la comunicación, constituye un requisito previo de muchas actividades sociales. Es tan importante la estructura de la interacción y de los encuentros sociales que si no existiera, afirma Goffman, la sociedad llegaría a disgregarse, porque es en las interacciones cara a cara donde el individuo aprende el sentido de la realidad social y donde se forma la concepción de su propio yo y del de los demás.

A finales de 1957, Herbert Blumer estaba montando, en la Universidad de California (en Berkeley), un Departamento con todas las tendencias teóricas y metodológicas de la sociología. Le falta alguien de la orientación psicosociológica, y el perfil de Goffman responde 
a lo que está buscando. Así, el 1 de enero de 1958, Goffman es contratado como «profesor ayudante visitante».

En 1963, Goffman publica Stigma. Notes on the Management of Spoiled Identity. En él, Goffman centra su atención en la gran diferencia que existe entre lo que un individuo debería ser, es decir, su «identidad social virtual», y lo que realmente es, es decir, su «identidad social real». Todo individuo que experimenta esa diferencia entre las dos identidades está estigmatizado.

Goffman considera detalladamente las presentaciones del yo de una variedad de personas, incluyendo los ciegos, los miembros de grupos minoritarios, los ex pacientes mentales, los ex presos, las mujeres, las personas obesas, los enanos, etc., que manejan, de una manera especial, las impresiones porque tienen la identidad deteriorada, es decir, son estigmatizados.

Además, en este libro se analiza la interacción dramática entre los individuos estigmatizados y los normales. Según Goffman, la naturaleza de esta interacción depende del tipo de estigma que posea el individuo. En el caso del estigma «desacreditado», el actor asume que las diferencias son evidentes y, por lo tanto, perceptibles para los miembros de la audiencia (por ejemplo, un parapléjico o un manco). Por el contrario, en el caso del estigma «desacreditable», las diferencias no son reconocibles ni perceptibles por la audiencia (por ejemplo, un individuo que ha tenido voluntariamente una experiencia homosexual). Para los individuos que poseen un estigma desacreditado, el problema dramático fundamental reside en manejar la tensión que conlleva el hecho de que los demás individuos se dan cuenta de que existe ese estigma, mientras que para los individuos que sufren un estigma desacreditable el problema dramático consiste en manejar la información de tal forma que su estigma siga siendo desconocido por la audiencia.

Gran parte del libro de Goffman trata de los individuos que tienen estigmas evidentes, y a veces grotescos (pérdida de la nariz, labio leporino, etc.), pero a medida que se avanza en su lectura se va haciendo patente lo que Goffman intenta transmitir y que es, que antes o después, en algún momento o lugar determinado, todos somos estigmatizados. Los ejemplos que cita Goffman incluyen al judío que vive en una comunidad predominantemente cristiana, a un individuo gordo entre un grupo de personas con pesos normales, y a la persona que ha mentido acerca de su pasado y se esfuerza constantemente en evitar que la audiencia pueda llegar a saberlo.

En 1968 deja Berkeley, donde era profesor titular desde 1960 y catedrático desde 1962, y se va a Filadelfia, donde la Universidad de Pennsylvania le había ofrecido una de sus pres- 
tigiosas cátedras «Benjamin Franklin». Lo único que tiene que hacer es dirigir un seminario para estudiantes de tercer ciclo, y vuelve a escribir.

En estos años, Goffman se interesa por el «orden público». Tradicionalmente, los sociólogos lo analizaban desde un punto de vista completamente diferente, centrándose en su alteración. El planteamiento de Goffman se basa en la observación de la conducta corriente y en el análisis de las reglas que los individuos siguen en la vida cotidiana.

Sostiene Goffman que los contactos habituales de las personas entre sí crean universalmente sus propias normas. Existen normas sobre la manera de comportarse en una calle llena de gente, sobre cuál es el momento adecuado para interpelar a un desconocido o sobre dónde colocarse en un ascensor casi lleno. Como del incumplimiento de estas normas el individuo no obtiene ningún beneficio, en general las cumple y confía en que los demás harán lo mismo. De esta manera, dichas reglas pasan a convertirse en supuestos semiconscientes. Si sabemos cuáles son algunos de estos supuestos, comprenderemos por qué mucha gente se siente muy incómoda si se transgreden ciertas normas públicas de comportamiento. Considerando, por ejemplo, la forma de vestirse, que es una elección personal, está claro que vestirnos como creemos que debemos hacerlo es una manera de manifestar respeto por una situación social concreta y por los individuos que forman parte de ella.

Todos somos conscientes de que vivimos en una comunidad donde, deliberadamente o no, se nos observa constantemente, de tal manera que, como actores sociales, siempre intentamos representar, lo mejor posible, nuestro papel. Por eso, Goffman analiza las relaciones que todo grupo de actores tiene normalmente entre sí, es decir, los aspectos de las relaciones sociales que se dan cuando los individuos se encuentran unos en presencia de otros. Lo que le interesa a Goffman son las normas y las prácticas que emplea cualquier participante concreto en el cauce de las relaciones mutuas; le interesan también las reglas y los ordenamientos conexos de conducta pertenecientes a la vida pública; le interesa, en concreto, el «orden público». Este interés por el orden público se centra en las situaciones en que los individuos pasan a ser físicamente accesibles a otros individuos. Como afirma Goffman, en estas situaciones el orden puede ser un asunto fundamental. Para estudiar todo esto, Goffman recurre a la observación no sistemática, naturalista.

En 1974 publica Frame Analisys, su gran libro de teoría que lleva diez años elaborando. El objetivo del libro, que se subtitula «Ensayo sobre la ordenación de la experiencia», es «intentar aislar algunos de los marcos de referencia disponibles en nuestra sociedad que son básicos para la comprensión y la explicación del sentido de los acontecimientos, así como analizar los riesgos especiales a que están sujetos esos marcos de referencia» (Goffman, 
1974: 8). Lo que Goffman pretende demostrar es que, al igual que en el largometraje de Truffaut «La noche americana», cualquier situación de la vida cotidiana es como una película dentro de otra porque las diferentes realidades se mezclan y confunden. "Frame Analysis es una obra fundamentalmente visual... es una magna obra dominada por la metáfora del cine, donde Goffman funde todos sus estudios anteriores» (Winkin, 1991: 84).

La pasión de Goffman por el cine también se hace patente en su libro Gender Advertisement, publicado en 1979 y que mereció el premio «George Orwell». Está plagado de fotos publicitarias que muestran las posturas físicas en que los medios de comunicación presentan siempre a la mujer. El libro es una reflexión y un análisis sobre el lugar que se otorga al género femenino en los anuncios publicitarios y en la sociedad occidental contemporánea. Está escrito con la misma rabia goffmaniana transformada en análisis frío que podemos encontrar en Internados y en Estigma y que conservará en Forms of Talk, su último libro.

Al parecer, y según sus biográfos, Goffman estaba en uno de sus mejores momentos. En 1981 se había casado con una lingüista de Montreal llamada Gillian Sankof y lo habían elegido Presidente de la Asociación Americana de Sociología. Unos meses más tarde, en mayo de 1982, antes de empezar su intervención en el coloquio «Cotidianeidad e Historicidad», organizado por la Universidad de Lyon II, dijo la que probablemente debe haber sido la única referencia a su vida privada: «Por causa de un suceso ajeno a mi voluntad —el nacimiento de mi hija— no he preparado ninguna comunicación» (Goffman, 1984: 197).

Después, todo será muy rápido y muy trágico. Lo hospitalizan al final del verano, por lo que tiene que anular el discurso que, como Presidente de la Asociación Estadounidense de Sociología, iba a pronunciar en San Francisco. Este discurso, titulado «El orden de la Interacción», es lo último que escribió Goffman. Poco después de redactarlo, en noviembre de 1982, Goffman ingresó en el hospital en el que moriría de cáncer días más tarde. Por ello nunca fue pronunciado pero, afortunadamente, la Asociación Americana de Sociología lo publicó en su revista un año después. Probablemente es, como afirma Yves Winkin, «un testamento en el que Goffman ha volcado toda su sociología, refutando a unos (a los que no quieren ver en la sociedad más que un conglomerado de interacciones) y alentando a otros (los que logran distinguir, pero vinculándolas, micro y macroestructuras)» (Winkin, 1991: 169).

En este artículo, Goffman define la interacción social como «aquella que se da exclusivamente en las situaciones sociales, es decir, en las que dos o más individuos se hallan en presencia de sus respuestas físicas respectivas» (Goffman, 1983: 2). Esto implica, sin embargo, aunque parezca contradictorio, que cierta disyuntiva sociológica fundamental puede no ser, en principio, relevante. Ésta es la diferenciación entre vida urbana y rural, entornos 
públicos y privados, relaciones íntimas de larga duración e impersonales y fugaces, etc. Al fin y al cabo, como afirma Goffman, las reglas de tráfico peatonales pueden analizarse de igual forma en una oficina llena de gente o en una calle abarrotada, las interrupciones verbales también pueden estudiarse durante una comida familiar o en una sesión parlamentaria, y las discusiones entre las parejas tanto en unos grandes almacenes como en la intimidad de su hogar.

Todo esto no es más que una forma de poner de manifiesto el empeño constante en la obra de Goffman de que sus observaciones sobre la vida cotidiana (que él llama «el orden de interacción») tuvieran la misma aceptación que cualquier otro tipo de análisis sociológico.

En cuanto a los procesos y estructuras concretos del orden de la interacción, Goffman argumenta que, probablemente, lo característico de la interacción cara a cara esté, de alguna manera, sometido al espacio y al tiempo. Como afirma Goffman, «la suspensión de una actividad interaccional que se ha iniciado tiene un efecto trascendental sobre ella y no se puede extender demasiado sin alterarla profundamente» (Goffman, 1983: 3). Es decir, en todo orden interaccional el nivel de compromiso y la concentración de los individuos es siempre crítico e impredecible, por lo que estos estados cognitivos no pueden mantenerse durante mucho tiempo. Parece claro que el estado de ánimo, la emoción, la cognición, la orientación corporal y el esfuerzo muscular están involucrados y suponen un elemento, tanto biológico como psicológico, insoslayable.

Goffman termina su discurso con una reivindicación fundamental: la del «oficio de sociólogo». Como dicen sus últimas palabras: «... Pero tenemos una cosa que no debemos cambiar por nada del mundo: la facilidad para mantener un espíritu libre e independiente frente a cualquier elemento de la vida social y la cordura para buscar sólo en nosotros y en nuestra disciplina esta aspiración. Ésta es nuestra herencia y lo que nosotros legaremos» (Goffman, 1983: 17).

Aunque la originalidad e importancia del modelo goffmaniano son reconocidas por casi todos los científicos sociales españoles, hay que decir que su pensamiento no ha tenido, en nuestro país, la penetración que hubiera sido deseable.

Mientras que en Estados Unidos su obra ha sido y es ampliamente comentada y analizada (la bibliografía en inglés es numerosísima) y en Europa podemos encontrar ensayos, cursos y seminarios sobre él en la Universidad Libre de Bruselas (Javeau, 1982 y 1984; Nahavandi, 1983), en Alemania (Müller, Schäfer, Kuzmics, Wehrspaunm, y la obra colectiva Erving Goffman, 1991), en el Centro de Estudios sobre lo Actual y lo Cotidiano de la Sor- 
bona con Maffesoli y Balandier, en Polonia (Czyzewski, 1981; Tittenbrun, 1981), en Italia (Wolf, 1979; Giglioli, 1984; Trifiletti, 1991), incluso en Japón y en la India (obra colectiva Beyond Goffman, 1991), en España - aunque es citado a menudo en manuales de sociología (Jiménez Blanco y Moya, 1978) y de psicología social (Torregrosa y Crespo, 1982; Munné, 1989; Rodríguez y Seoane, 1989; Jiménez Burillo, 1991) - se pueden contar con los dedos las reflexiones teóricas que, en forma de artículo o de libro, existen sobre su pensamiento. Entre otros, hay que citar el artículo del profesor J. J Caballero Romero «La interacción social en Goffman», y el libro de Sebastián de Erice Erving Goffman: De la interacción focalizada al orden interaccional. Este autor afirma que en España se conoce a Goffman desde la presentación que hizo Amando de Miguel en Introducción a la Sociología de la vida cotidiana, en 1969, y profesores de la talla de J. L. López Aranguren y Gutiérrez López han impartido seminarios sobre su obra en la Universidad Complutense y en la de Granada. También sociólogos como Garmendia han presentado su obra en la revista Debats (vol. 19, 1984), con artículos de Picó, Rivière y Crespi, y en estos momentos se pueden encontrar cursos de Doctorado en algunas Facultades de la Universidad Complutense.

Sin embargo, si tenemos en cuenta que han transcurrido treinta y tres años desde la presentación de A. de Miguel y veinte de la muerte de Goffman, hay que reconocer el escaso eco que, en nuestro país, ha tenido este microsociólogo, quizá porque en nuestro panorama sociológico todavía se considera lo «micro» —que se ocupa de lo obvio, lo aparentemente irrelevante, lo difícil de cuantificar - como opuesto a lo «macro» —que se interesa por lo serio, lo relevante, lo importante, lo medible-, es decir, la Sociología con mayúsculas, o quizá porque en la psicología social española — donde creo que es el mejor lugar para ubicar a Goffman - ha dominado y sigue dominando hoy en día el hegemónico paradigma conductista. Sea cual sea la causa, habría que pasar página e impulsar en todos los campos de las ciencias sociales un minucioso estudio de este autor, que tiene, todavía, mucho que aportar. Valgan estas líneas como estímulo y reivindicación.

\section{BIBLIOGRAFÍA}

BERGER, P., y LUCKMANN, T. (1966): The social construction of Reality, Doubleday, New York. Trad. esp.: La construcción social de la realidad, Amorrortu, Buenos Aires, 1968.

BOLTANSKI, L. (1973): «Erving Goffman et le temps du soupçon», Information sur les Sciences Sociales, 12-3.

BURNS, T. (1992): Erving Goffman, Routledge, London and New York.

CABALLERO ROMERO, J. J. (1998): «La interacción social en Goffman», REIS, 83, pp. 121-149.

CZYZEWSKI, M. (1981): «Miejsce analizy ramowej w socjiologii Ervinga Goffmana», Przeglad Socjiologiczny, 33, pp. 195-215. 
DREW, P., y WOOTTON, A. (comps.) (1988): Erving Goffman: Exploring the interaction order, Northeastern University Press, Boston.

- (comps.) (1989): Erving Goffman: An interdisciplinary appreciation, Polity Press, Cambridge.

GOFFMAN, E. (1949): Some Characteristics of Response to Depicted Experience. A dissertation submitted to the Faculty of the Division of the Social Sciences in candidacy for the Degree of Master of Arts, Universidad de Chicago, Departamento de Sociología, diciembre.

- (1953): Communication Conduct in a Island Community. A Dissertation submitted to the Faculty of the Division of the Social Sciences in Candidacy for the Degree of Doctor of Philosophy, Universidad de Chicago, Departamento de Sociología.

- (1957): Characteristics of Total Institutions, U.S. Army, Symposium on Preventive and Social Psychiatry.

- (1959): Presentation of Self in Everyday Life, Anchor Books, New York. Trad. esp.: La presentación de la persona en la vida cotidiana, Amorrortu, Buenos Aires, 1971.

- (1961): Asylums. Essays on the Social Situation of Mental Patients and Other Inmates, Anchor Books, New York. Trad. esp.: Internados. Ensayos sobre la situación social de los enfermos mentales, Amorrortu, Buenos Aires, 1971.

— (1964): Stigma, Prentice-Hall, Inc., Englewod Cliffs (NJ). Trad. esp.: Estigma, Amorrortu, Buenos Aires, 1970.

- (1967): Interaction Ritual, Anchor Books, New York. Trad. esp.: Ritual de la Interacción, Tiempo Contemporáneo, Buenos Aires, 1970.

- (1971): Relation in Public, Basic Books (Paper-back. Version Harper and Row), New York. Trad. esp.: Relaciones en Público, Alianza, Madrid, 1979.

- (1974): Frame Analysis. An Essay on the Organization of Experience, Harper and Row, New York. Traduccióm inédita de J. L. Rodríguez: Análisis del Marco Social. Ensayo sobre la ordenación de la experiencia.

- (1979): Gender Advertisements, Harper and Row (George Orwell Award), New York.

— (1983): «The Interaction Order», American Sociological Review, vol. 48, n. ${ }^{\circ}$ 1, febrero.

— (1984): «Microsociologie et histoire», en Ph. Fritsch (comp.), Le Sens de l'Ordinaire, Presses Universitaires de Lyon, Lyon.

GIGLIOLI, P. (1984): «Una lettura durkheimiana di Goffman», Rassegna Italiana di Sociologia, 25, pp. 401-427.

HETTLAGE, R., y LENZ, K. (comps.) (1991): Erving Goffman: ein zociologischer Klassiker der zweiten Generation, Paul Haupt, Berna y Stuttgart.

JAVEAU, C. (1982): «Pour une sociologie descriptive de la vie quotidienne: quelques pistes et quelques détours», Recherches Sociologiques, 13, pp. 27-38.

- (1984): «Diderot, Goffman et le mesonge social», Revue Internationale de Philosophie, 38, pp. 171-181.

JIMÉNEZ BLANCO, J., y MOYA, C. (comps.) (1978): Teoría Sociológica Contemporánea, Tecnos, Madrid.

JIMÉNEZ BURILLO, F. (1991): Psicología Social, Ediciones Académicas, Madrid.

JOSEPH, I., et al. (1989): Le parler frais d'Erving Goffman, Les Editions de Minuit, París.

KUZMICS, H. (1986): «Verlegenheit und Zivilisation. Zu einigen Gemeinsamkeiten und Unterschieden im Work von E. Goffman und N. Elias», Soziale Welt, 37, pp. 465-486. 
LOFLAND, J. (1980): «Early Goffman: Style, Structure, Substance, Soul», en Jason Ditton (ed.), The View from Goffman, St. Martin's Press, New York, p. 47.

MARIANINI, P. (1972): La società e le cose. Sociologia e ideologia da Durkheim a Goffman, Istituto Librario Internazionale, Milán.

NAHAVANDI, F. (1983): «Les sociologues américains et la sociologie de la vie quotidienne», en C. Javeau (comp.), Micro et macro-sociologie de la vie quotidienne, Institut de Sociologie, Bruselas, pp. 65-66.

MIGUEL, A. de (1969): Introducción a la sociología de la vida cotidiana, Cuadernos para el Diálogo, Madrid.

MÜLLER, G. (1983): «Kritische Bemerkungrn zum Begriff Rollendistanz in der gegenwärtigen Soziologie-dargestellt am Beispiel E. Goffman», Wissenschaftliche Zeitschrift der Karl Marx Universität Leipzig, 32, pp. 314-318.

MUNNÉ, F. (1989): Entre el individuo y la sociedad. Marcos y teorías actuales sobre el comportamiento interpersonal, Biblioteca Universitaria de Ciencias Sociales, Barcelona.

RIGGIN, S. (comp.) (1990): Beyond Goffman: Studies on communication, institution, and social interaction, Mouton de Gruyter, Berlín y Nueva York.

RITZER, G. (1993): Teoría Sociológica Contemporánea, McGraw-Hill, Madrid.

RODRÍGUEZ, A., y SEOANE, J. (1989): «Creencias, actitudes y valores», en J. Mayo y J. L. Pinillos (comps.), Tratado de Psicología General, Alhambra Universidad, Madrid.

SCHÄFER, S. (1983): «Identität und sekundäre Anpassung. Zum theoretischen Bezugsrahmen Erving Goffman», Kölner Zeitschrift für Soziologie und Sizialpsychologie, 35, pp. 631-654.

SEBASTIÁN DE ERICE, J. (1994): Erving Goffman. De la interacción focalizada al orden interaccional, CIS, Madrid.

TITTENBRUN, J. (1981): “Czlowiek, interkeja, spoleczenstwo (o teorii Ervinga Goffmana)», Kultura i Spoleczenstwo, 25, pp. 291-301.

TORREGROSA, J. R., y CRESPO, E. (1982): Estudios básicos de Psicología Social, Hora, Barcelona.

TRIFILETTI, E. (1991): L'identità controversa. L'itinerario di Erving Goffman nella sociologia contemporanea, Cedam, Padua.

WEHRSPAUN, M. (1989): «Kommunikation, öffenliche Ordnung und das projektive Selbst. Die Bedeutung von Erving Goffman. Ökologie der sozialen Situation für die Analyse der Moderne», Zeitschrift für Soziologie, 18, pp. 329-345.

WINKIN, Y. (1988): Les moments et leur hommes, Les Éditions du Seuil, París. Trad. esp.: Los momentos y sus hombres, Paidós, Barcelona, 1991.

WOLF, M. (1979): Sociologie della vita quotidiana, L’Esspreso. Trad. esp.: Sociologías de la vida cotidiana, Cátedra, Madrid. 
ABSTRACT

Although Erving Goffman's work has still low implantation in spanish academic sociology, there is no doubt that he is one of the most important and original microsociologists in the second half of last century. That is why, in the twentieth anniversary of his death and eight years after his birth, I have tried to offer a brief biographical and bibliographical revision of his life and his thought. Educated in the Symbolic Interactionist School and creator of the Dramaturgical Model his scientific interests makes him analyse research areas such as interaction in everyday life, the social construction of the stigma, the identity and the self, the Total Institutions, etc., wich are topical problems in the current psychosociologyst discussions. 